\title{
A Common Fixed Point Theorem in Fuzzy Metric Spaces with Nonlinear Contractive Type Condition Defined Using $\Phi$-Function
}

\author{
Siniša N. Ješić, ${ }^{1}$ Nataša A. Babačev, ${ }^{1}$ and Rale M. Nikolić ${ }^{2}$ \\ ${ }^{1}$ Department of Applied Mathematics, Faculty of Electrical Engineering, Bulevar Kralja Aleksandra 73, \\ P.O. Box 35-54, 11120 Beograd, Serbia \\ ${ }^{2}$ Faculty of Information Technology, Metropolitan University, Tadeuša Košćuška 63, 11000 Belgrade, Serbia \\ Correspondence should be addressed to Siniša N. Ješić; jesha@eunet.rs
}

Received 11 November 2012; Accepted 30 January 2013

Academic Editor: Fasma Diele

Copyright (c) 2013 Siniša N. Ješić et al. This is an open access article distributed under the Creative Commons Attribution License, which permits unrestricted use, distribution, and reproduction in any medium, provided the original work is properly cited.

This paper is to present a common fixed point theorem for two $R$-weakly commuting self-mappings satisfying nonlinear contractive type condition defined using a $\Phi$-function, defined on fuzzy metric spaces. Some comments on previously published results and some examples are given.

\section{Introduction and Preliminaries}

The theory of fuzzy sets was introduced by Zadeh in [1]. Definitions of fuzzy metric spaces were given by Kaleva and Seikkala in [2] and by Kramosil and Michálek in [3], and these definitions are accepted by many mathematicians and physicists. The modification of these definitions were given by George and Veeramani in [4].

Definition 1 (see [5]). A binary operation $*:[0,1] \times[0,1] \rightarrow$ $[0,1]$ is a continuous $t$-norm if $([0,1], *)$ is a topological monoid with unit 1 such that $a * b \leq c * d$ whenever $a \leq c$ and $b \leq d$ are satisfied, for all $a, b, c, d \in[0,1]$.

Example 2. $a * b=a b, a * b=\min \{a, b\}$.

Definition 3 (see [1]). A fuzzy set $A$ in $X$ is a function with domain in $X$ and values in $[0,1]$.

Definition 4 (see [3]). The 3-tuple $(X, M, *)$ is said to be a fuzzy metric space in the sense of Kramosil and Michálek, where $X$ is an arbitrary set, $*$ is a continuous $t$-norm, and $M$ is a fuzzy set defined on set $X^{2} \times[0, \infty)$ satisfying the following conditions:

$(\mathrm{FM}-1) M(x, y, 0)=0$,
(FM-2) $M(x, y, t)=1$, for every $t>0$ if and only if $x=y$,

(FM-3) $M(x, y, t)=M(y, x, t)$, for all $x, y \in X$ and $t>0$,

(FM-4) $M(x, y, t) * M(y, z, s) \leq M(x, z, t+s)$, for all $x, y, z \in$ $X$ and $t, s>0$,

$(\mathrm{FM}-5) M(x, y, \cdot):(0, \infty) \rightarrow[0,1]$ is a left continuous function, for all $x, y \in X$.

A function $M$ is called a fuzzy metric.

Remark 5 (see [4]). Let $(X, d)$ be a metric space and $a * b=a b$ and

$$
M(x, y, t)=\frac{k t^{n}}{k t^{n}+m \cdot d(x, y)},
$$

for $k, m, n \in \mathbb{R}^{+}$. Then, $(X, M, *)$ is a fuzzy metric space. The 3-tuple $(X, M, *)$ is a fuzzy metric space, also, if we observe a $t$-norm $a * b=\min \{a, b\}$ instead of $a * b=a b$. Taking that $k=m=n=1$ in (1), we get

$$
M(x, y, t)=\frac{t}{t+d(x, y)} .
$$

A fuzzy metric given by (2) is called the standard fuzzy metric, and it is induced by a metric $d$. 
Grabiec in [6] proved the next lemma.

Lemma 6 (see [6]). Let $(X, M, *)$ be a fuzzy metric space. Then, $M(x, y, \cdot)$ is a nondecreasing function, for all $x, y \in X$.

Definition 7 (see [4]). A sequence $\left\{x_{n}\right\}$ in a fuzzy metric space $(X, M, *)$ is said to converge to $x \in X$ if and only if for every $\varepsilon>0$ and $t>0$, there exists $n_{0} \in N$ such that $M\left(x_{n}, x, t\right)>$ $1-\varepsilon$, for every $n>n_{0}$, that is, $\lim _{n \rightarrow \infty} M\left(x_{n}, x, t\right)=1$, for every $t>0$. In that case, write one $\lim _{n \rightarrow \infty} x_{n}=x$.

Definition 8 (see [4]). A sequence $\left\{x_{n}\right\}$ in a fuzzy metric space $(X, M, *)$ is said to be a Cauchy sequence if for every $\varepsilon>0$ and $t>0$, there exists $n_{0} \in \mathbb{N}$ such that $M\left(x_{n}, x_{m}, t\right)>1-\varepsilon$, for all $n, m>n_{0}$. A fuzzy metric space is said to be complete if every Cauchy sequence is convergent.

The following lemma is essentially given in [6].

Lemma 9. Let $(X, M, *)$ be a fuzzy metric space and $\lim _{n \rightarrow \infty} x_{n}=x$ and $\lim _{n \rightarrow \infty} y_{n}=y$. Then, for every fixed $t>0$, it holds

$$
\liminf _{n \rightarrow \infty} M\left(x_{n}, y_{n}, t\right)=M(x, y, t) .
$$

George and Veeramani in [4] introduced Hausdorff topology in fuzzy metric spaces. This topology is first countable.

Definition 10 (see [4]). Let $(X, M, *)$ be a fuzzy metric space. Open ball $B(x, r, t)$ with center in $x \in X$, and radius $r \in(0,1)$, for $t>0$ is defined as follows:

$$
B(x, r, t)=\{y \in X \mid M(x, y, t)>1-r\} .
$$

Topology in fuzzy metric spaces is defined as follows:

$\tau=\{A \subset X \mid x \in A$ if and only if there exists $t>0$,

$$
r \in(0,1) \text {, such that } B(x, r, t) \subset A\} .
$$

Theorem 11 (see [4]). Every open ball is an open set.

Theorem 12 (see [4]). Every fuzzy metric space is Hausdorff.

Definition 13. Let $(X, M, *)$ be a fuzzy metric space and $A \subseteq$ $X$. The closure of the set $A$ is the smallest closed set containing $A$, denoted by $\bar{A}$.

Obviously, having in mind the Hausdorff topology and the definition of converging sequences, we have that the next remark holds.

Remark 14. $x \in \bar{A}$ if and only if there exists a sequence $\left\{x_{n}\right\}$ in $A$ such that $x_{n} \rightarrow x$.

The facts that will play the important role in the proof of the main result are the following definition and theorem which are given and proved in [7].

Definition 15 (see [7]). Let $(X, M, *)$ be a fuzzy metric space. A collection $\left\{F_{n}\right\}_{n \in \mathbb{N}}$ is said to have fuzzy diameter zero if for each $r \in(0,1)$ and each $t>0$, there exists $n_{0} \in \mathbb{N}$ such that $M(x, y, t)>1-r$, for all $x, y \in F_{n_{0}}$.

Theorem 16 (see [7]). A fuzzy metric space $(X, M, *)$ is complete if and only if every nested sequence $\left\{F_{n}\right\}_{n \in \mathbb{N}}$ of nonempty closed sets with fuzzy diameter zero has nonempty intersection.

Remark 17 (see [7]). The element $x \in \bigcap_{n \in \mathbb{N}} F_{n}$ is unique.

The concept of bounded sets in probabilistic metric spaces is very close to the concept of strong boundness in fuzzy metric spaces.

Definition 18. Let $(X, M, *)$ be a fuzzy metric space. Let the mappings $\delta_{A}(t):(0, \infty) \rightarrow[0,1]$ be defined as

$$
\delta_{A}(t)=\inf _{x, y \in A} \sup _{\varepsilon<t} M(x, y, \varepsilon) .
$$

The constant $\delta_{A}=\sup _{t>0} \delta_{A}(t)$ will be called fuzzy diameter of set $A$. If $\delta_{A}=1$ the set $A$ will be called $F$-strongly bounded.

Lemma 19. Let $(X, M, *)$ be a fuzzy metric space. A set $A \subseteq X$ is an F-strongly bounded if and only if for each $r \in(0,1)$, there exists $t>0$ such that $M(x, y, t)>1-r$, for all $x, y \in A$.

Proof. The proof follows from the definitions of $\sup A$ and inf $A$ of nonempty sets.

Example 20. Let $(X, M, *)$ be a fuzzy metric space induced by a metric $d$ on $X$ given in Remark 5. $A \subseteq X$ is metrically bounded if and only if it is an F-strongly bounded.

Proof. Let $A \subseteq X$ be metrically bounded, that is, $d(x, y)<k$, for some $k \in \mathbb{R}$ and all $x, y \in A$. Let $r \in(0,1)$ be arbitrary. We will prove that there exists $t>0$ such that $M(x, y, t)>1-r$, for all $x, y \in A$. Let us take $t$ such that $t>k(1-r) / r$. For this $t$, it follows that $t r>k(1-r)$ from which it follows that $0>k(1-r)-t r$. Finally, from the last inequality, we get that $t>k(1-r)+t-t r$, that is, $t /(t+k)>1-r$. Since $d(x, y)<k$, for all $x, y \in A$, it follows that $M(x, y, t)=t /(t+d(x, y))>$ $t /(t+k)>1-r$, for all $x, y \in A$. Applying Lemma 19, we get that $A$ is an $F$-strongly bounded.

Conversely, if $A$ is an $F$-strongly bounded set, then for arbitrary $r \in(0,1)$, there exists $t>0$ such that $M(x, y, t)=$ $t /(t+d(x, y))>1-r$, for all $x, y \in A$. From this inequality, it follows that $d(x, y)<r t /(1-r)$, for all $x, y \in A$, that is, the set $A$ is metrically bounded. This completes the proof.

Properties of fuzzy metric spaces and fixed point results in these spaces are obtained by several authors $[4,7-11]$. Results presented in $[10,11]$ consider the fixed point results for mappings satisfying nonlinear contractive type condition, but the class of fuzzy metric spaces is restrictive because the authors added an additional axiom for fuzzy metric spaces. Our result is applicable for arbitrary fuzzy metric space without restrictions. Recently, Miheţ in [12] proved a fixed point theorem for mappings defined on probabilistic metric spaces satisfying nonlinear contractive type condition 
defined using a $\Phi$-function. We will prove a common fixed point theorem for mappings defined on fuzzy metric spaces satisfying nonlinear contractive type condition defined using $\Phi$-function.

Khan et al. in [13] introduced the concept of altering distance functions that alter the distance between two points in metric spaces.

Definition 21 (see [13]). A function $h:[0, \infty) \rightarrow[0, \infty)$ is an altering distance function if

(i) $h$ is monotone increasing and continuous,

(ii) $h(t)=0$ if and only if $t=0$.

Choudhury and Das [14] extended the concept of altering distance functions to Menger PM-spaces.

Definition 22 (see [14]). A function $\phi:[0, \infty) \rightarrow[0, \infty)$ is said to be a $\Phi$-function if the following conditions hold

(i) $\phi(t)=0$ if and only if $t=0$,

(ii) $\phi$ is strictly increasing and $\phi(t) \rightarrow \infty$ as $t \rightarrow \infty$,

(iii) $\phi$ is left continuous in $(0, \infty)$,

(iv) $\phi$ is continuous at 0 .

The class of all $\Phi$-functions will be denoted by $\Phi$.

Choudhury and Das [14] proved the following result.

Theorem 23 (see [14]). Let $\left(X, \mathscr{F}, T_{M}\right)$ be a complete Menger PM-space, with continuous t-norm $T_{M}$ given by $T_{M}(a, b)=$ $\min \{a, b\}$, and let $f$ be a continuous self-mapping on $X$ such that for every $x, y \in X$, and all $t>0$ holds

$$
F_{f x, f y}(\phi(t)) \geq F_{x, y}\left(\phi\left(\frac{t}{c}\right)\right),
$$

where $\phi$ is a $\phi$-function and $0<c<1$. Then, $f$ has a unique fixed point. in $[12]$.

The previous result is generalized and improved by Miheţ

Theorem 24 (see [12]). Let $(X, \mathscr{F}, T)$ be a complete Menger $P M$-space with continuous t-norm $T$, and let $f$ be a selfmapping on $X$ such that for every $x, y \in X$, and all $t>0$ holds

$$
F_{f x, f y}(\phi(t)) \geq F_{x, y}\left(\phi\left(\frac{t}{c}\right)\right)
$$

where $\phi$ is a $\Phi$-function and $0<c<1$. If there exists $x \in X$ such that the orbit of $f$ in $x, \mathcal{O}(f, x)=\left\{f^{m} x, m \in \mathbb{N} \backslash\{0\}\right\}$ is probabilistic bounded, then $f$ has a unique fixed point.

By proving common fixed point results, several authors observed different generalizations of commutativity. The concept of $R$-weakly commuting mappings was introduced by Pant in [15]. Following Pant, the definition of $R$ weakly commutativity for mappings defined on spaces with nondeterministic distances was given in $[8,9,16]$.
Definition 25. Let $(X, M, *)$ be a fuzzy metric space, and let $f$ and $g$ be self-mappings of $X$. The mappings $f$ and $g$ will be called $R$-weakly commutings, if there exists some positive real number $R$ such that

$$
M(f(g(x)), g(f(x)), R t) \geq M(f(x), g(x), t),
$$

for all $t>0$ and each $x \in X$.

\section{Main Results}

Lemma 26. Let $(X, M, *)$ be a fuzzy metric space. Let $\phi$ be a $\Phi$-function and $0<c<1$. If for $x, y \in X$, it holds that

$$
M(x, y, \phi(t)) \geq M\left(x, y, \phi\left(\frac{t}{c}\right)\right)
$$

for all $t>0$, then $x=y$.

Proof. Let us suppose that $M(x, y, \phi(t)) \geq M(x, y, \phi(t / c))$ and $x \neq y$. From this condition, we have $M(x, y, \phi(t)) \leq$ $M(x, y, \phi(c t))$ and, by induction, we have $M(x, y, \phi(t)) \leq$ $M\left(x, y, \phi\left(c^{n} t\right)\right)$. Taking limit as $n \rightarrow \infty$, we get that $M(x, y, \phi(t))=0$, for all $t>0$, which is a contradiction, that is, $x=y$.

Theorem 27. Let $(X, M, *)$ be a complete fuzzy metric space. Let $f$ and $g$ be R-weakly commuting self-mappings on $X$, and let $f$ be continuous such that $g(X) \subseteq f(X)$. Let, for all $x, y \in X$ and every $t>0$,

$$
M(g(x), g(y), \phi(t)) \geq M\left(f(x), f(y), \phi\left(\frac{t}{c}\right)\right)
$$

hold, where $\phi$ is a $\Phi$-function and $0<c<1$. If there exists $a$ point $u_{0} \in X$ and $n_{0} \in \mathbb{N}$ such that the set

$$
S=\left\{g\left(u_{n_{0}-1}\right), g\left(u_{n_{0}}\right), \ldots\right\}
$$

where $f\left(u_{i}\right)=g\left(u_{i-1}\right)(i \in \mathbb{N})$ is an F-strongly bounded set, then the mappings $g$ and $f$ have a unique common fixed point in $X$.

Proof. First, we will prove that the mapping $g$ is continuous. We will prove that for sequence $y_{n} \rightarrow y$, it follows that $g\left(y_{n}\right) \rightarrow g(y)$ as $n \rightarrow \infty$. Let $t>0$ be arbitrary. For arbitrary $t>0$, there exists $s>0$ such that $t>\phi(s)$. Since $f$ is continuous, it follows that $f\left(y_{n}\right) \rightarrow f(y)$, that is, for all $t>0$, it holds that $M\left(f\left(y_{n}\right), f(y),(t)\right) \rightarrow 1$ as $n \rightarrow \infty$. Since the function $M(x, y, \cdot)$ is nondecreasing for all $x, y \in X$, applying (11), we have

$$
\begin{aligned}
M\left(g\left(y_{n}\right), g(y), t\right) & \geq M\left(g\left(y_{n}\right), g(y), \phi(s)\right) \\
& \geq M\left(f\left(y_{n}\right), f(y), \phi\left(\frac{s}{c}\right)\right) \longrightarrow 1,
\end{aligned}
$$

as $n \rightarrow \infty$, that is, $M\left(g\left(y_{n}\right), g(y), t\right) \rightarrow 1$ as $n \rightarrow \infty$, for all $t>0$, that is, $g\left(y_{n}\right) \rightarrow g(y)$ as $n \rightarrow \infty$.

For $u_{0} \in X$ from $g(X) \subseteq f(X)$, it follows that there exists a point $u_{1} \in X$ such that $g\left(u_{0}\right)=f\left(u_{1}\right)$. By induction, a 
sequence $\left\{u_{n}\right\}$ can be chosen such that $g\left(u_{n-1}\right)=f\left(u_{n}\right)$, and the set $S=\left\{g\left(u_{n_{0}-1}\right), g\left(u_{n_{0}}\right), \ldots\right\}$ is an $F$-strongly bounded.

Let us consider nested sequence of nonempty closed sets defined by

$$
\begin{array}{r}
F_{n}=\overline{\left\{g\left(u_{n-1}\right), g\left(u_{n}\right), \ldots\right\}}=\overline{\left\{f\left(u_{n}\right), f\left(u_{n+1}\right), \ldots\right\}}, \\
n \geq n_{0} .
\end{array}
$$
zero.

We will prove that the family $\left\{F_{n}\right\}_{n \geq n_{0}}$ has fuzzy diameter

Let $r \in(0,1)$ and $t>0$ be arbitrary. We will prove that there exists $n^{*} \in \mathbb{N}$ such that $M(x, y, t)>1-r$, for all $x, y \in$ $F_{n^{*}}$. For arbitrary $k \geq n_{0}, k \in \mathbb{N}$, from $F_{k} \subseteq \bar{S}$, it follows that $F_{k}$ is an $F$-strongly bounded set, that is, there exists $t_{0}>0$ such that

$$
M\left(x, y, t_{0}\right)>1-r \quad \forall x, y \in F_{k} .
$$

Since $\phi$ is a $\Phi$-function, it follows that there exists $l \in \mathbb{N}$ such that $\phi\left(t / c^{l}\right)>t_{0}$. Let $n^{*}=l+k$ and $x, y \in F_{n^{*}}$ be arbitrary. There exist sequences $\left\{g\left(u_{n(i)-1}\right)\right\},\left\{g\left(u_{n(j)-1}\right)\right\}$ in $F_{n^{*}}\left(n(i), n(j) \geq n^{*}, i, j \in \mathbb{N}\right)$ such that $\lim _{n(i) \rightarrow \infty} g\left(u_{n(i)-1}\right)=$ $x$ and $\lim _{n(j) \rightarrow \infty} g\left(u_{n(j)-1}\right)=y$.

Since for arbitrary $t>0$, there exists $s>0$ such that $t>$ $\phi(s)$, from (11), we have

$$
\begin{aligned}
& M\left(g\left(u_{n(i)-1}\right), g\left(u_{n(j)-1}\right), t\right) \\
& \quad \geq M\left(g\left(u_{n(i)-1}\right), g\left(u_{n(j)-1}\right), \phi(s)\right) \\
& \quad \geq M\left(f\left(u_{n(i)-1}\right), f\left(u_{n(j)-1}\right), \phi\left(\frac{s}{c}\right)\right) \\
& \quad=M\left(g\left(u_{n(i)-2}\right), g\left(u_{n(j)-2}\right), \phi\left(\frac{s}{c}\right)\right) .
\end{aligned}
$$

Thus, by induction, we get

$$
\begin{aligned}
& M\left(g\left(u_{n(i)-1}\right), g\left(u_{n(j)-1}\right), t\right) \\
& \quad \geq M\left(g\left(u_{n(i)-l-1}\right), g\left(u_{n(j)-l-1}\right), \phi\left(\frac{t}{c^{l}}\right)\right) .
\end{aligned}
$$

Since $\phi\left(t / c^{l}\right)>t_{0}$ and because $M(x, y, \cdot)$ is a nondecreasing function, from previous inequalities, it follows that

$$
\begin{aligned}
& M\left(g\left(u_{n(i)-1}\right), g\left(u_{n(j)-1}\right), t\right) \\
& \quad \geq M\left(g\left(u_{n(i)-l-1}\right), g\left(u_{n(j)-l-1}\right), \phi\left(\frac{t}{c^{l}}\right)\right) \\
& \quad \geq M\left(g\left(u_{n(i)-l-1}\right), g\left(u_{n(j)-l-1}\right), t_{0}\right) .
\end{aligned}
$$

As $\left\{g\left(u_{n(i)-l-1}\right)\right\}$ and $\left\{g\left(u_{n(j)-l-1}\right)\right\}$ are sequences in $F_{k}$ from (15) and (18), it follows that, for all $i, j \in \mathbb{N}$, it holds

$$
M\left(g\left(u_{n(i)-1}\right), g\left(u_{n(j)-1}\right), t\right)>1-r .
$$

Taking lim inf as $n(i), n(j) \rightarrow \infty$ and applying Lemma 9, we get that $M(x, y, t)>1-r$, for all $x, y \in F_{n^{*}}$, that is, the family $\left\{F_{n}\right\}_{n \geq n_{0}}$ has fuzzy diameter zero.
Applying Theorem 16, we conclude that this family has nonempty intersection, which consists of exactly one point $z$. Since the family $\left\{F_{n}\right\}_{n \geq n_{0}}$ has fuzzy diameter zero and $z \in F_{n}$, for all $n \geq n_{0}$, then for each $r \in(0,1)$ and each $t>0$ there exists $k_{0} \geq n_{0}$ such that for all $n \geq k_{0}$ holds

$$
M\left(g\left(u_{n-1}\right), z, t\right)>1-r .
$$

From the last, it follows that for each $r \in(0,1)$ and each $t>0$ holds

$$
\liminf _{n \rightarrow \infty} M\left(g\left(u_{n-1}\right), z, t\right)>1-r .
$$

Taking that $r \rightarrow 0$, we get that for each $t>0$, it holds that

$$
\liminf _{n \rightarrow \infty} M\left(g\left(u_{n-1}\right), z, t\right)=1 \text {, }
$$

that is, $\lim _{n \rightarrow \infty} g\left(u_{n-1}\right)=z$. From the definition of $\left\{f\left(u_{n}\right)\right\}$, we have $\lim _{n \rightarrow \infty} f\left(u_{n}\right)=z$.

Since $f$ and $g$ are $R$-weakly commutings, we have that for each $t>0$ it holds that

$$
M\left(f\left(g\left(u_{n}\right)\right), g\left(f\left(u_{n}\right)\right), t\right) \geq M\left(f\left(u_{n}\right), g\left(u_{n}\right), \frac{t}{R}\right) .
$$

Taking $\lim$ inf as $n \rightarrow \infty$ from previous inequality, for each $t>0$, it holds that

$$
M(f(z), g(z), t) \geq M\left(z, z, \frac{t}{R}\right)=1,
$$

that is, it holds that

$$
g(z)=f(z) .
$$

Let us prove that $f(z)=z$. From (11), it follows that for every $t>0$, it holds that

$$
\begin{aligned}
M & \left(g\left(f\left(u_{n}\right)\right), g\left(u_{n}\right), \phi(t)\right) \\
& \geq M\left(f\left(f\left(u_{n}\right)\right), f\left(u_{n}\right), \phi\left(\frac{t}{c}\right)\right) .
\end{aligned}
$$

Taking $\lim \inf$ as $n \rightarrow \infty$, we have that $M(g(z), z, \phi(t)) \geq$ $M(f(z), z, \phi(t / c))$ holds, for every $t>0$. Since $f(z)=g(z)$, it follows that

$$
M(f(z), z, \phi(t)) \geq M\left(f(z), z, \phi\left(\frac{t}{c}\right)\right) .
$$

Applying Lemma 26, we get that $f(z)=z$, that is, $z$ is a fixed point of $f$. From (25), it follows that $g(z)=z$, that is, $z$ is a common fixed point of $f$ and $g$.

Let us prove that $z$ is a unique common fixed point. For this purpose, let us suppose that there exists another common fixed point, denoted by $u$. From the starting condition, follow

$$
M(g(z), g(u), \phi(t)) \geq M\left(f(z), f(u), \phi\left(\frac{t}{c}\right)\right),
$$

for every $t>0$. Therefore, we get that

$$
M(z, u, \phi(t)) \geq M\left(z, u, \phi\left(\frac{t}{c}\right)\right),
$$

for every $t>0$. Finally, applying Lemma 26, it follows that $z=u$. This completes the proof. 
If we take that $f$ is identical mapping, in the statement of Theorem 27, since identical mappings commute with $f$ and commuting mappings are $R$-weakly commutings, we get the following theorem.

Theorem 28. Let $(X, M, *)$ be a complete fuzzy metric space. Let $g$ be a self-mapping on $X$ such that for all $x, y \in X$ and every $t>0$, it holds

$$
M(g(x), g(y), \phi(t)) \geq M\left(x, y, \phi\left(\frac{t}{c}\right)\right),
$$

where $\phi$ is a $\Phi$-function and $0<c<1$. If there exists a point $u_{0} \in X$ and $n_{0} \in \mathbb{N}$ such that the set

$$
S=\left\{g\left(u_{n_{0}-1}\right), g\left(u_{n_{0}}\right), \ldots\right\}
$$

where $u_{i}=g\left(u_{i-1}\right)(i \in \mathbb{N})$ is an F-strongly bounded set, then the mapping $g$ has a unique common fixed point in $X$.

Since every Menger probabilistic metric space is fuzzy metric space, Theorem 28 is an improvement of main result presented in [12].

Example 29. Let $(X, M, *)$ be a complete fuzzy metric space induced by the metric $d(x, y)=|x-y|$ on $X=[0,+\infty) \subset \mathbb{R}$ given in Remark 5. Let $f(x)=4 x, g(x)=4 x /(4+x), g(X)=$ $[0,4) \subset X=f(X), \phi(t)=t m$ and $c=1 / 4$.

We will prove that all the conditions of Theorem 27 are satisfied. Because

$$
f(g(x))=\frac{16 x}{4+x}, \quad g(f(x))=\frac{4 x}{1+x}
$$

we conclude that $f(x)$ and $g(x)$ are not commuting mappings. On the other hand, we have that

$$
\begin{gathered}
M(f(g(x)), g(f(x)), t)=\frac{t}{t+\left(12 x^{2} /(4+x)(1+x)\right)}, \\
M(f(x), g(x), t)=\frac{t}{t+\left(\left(4 x^{2}+12\right) /(4+x)\right)} .
\end{gathered}
$$

Since $12 x^{2} /(4+x)(1+x) \leq\left(4 x^{2}+12 x\right) /(1+x)$, for every $x \geq 0$, we have

$$
M(f(g(x)), g(f(x)), t) \geq M(f(x), g(x), t),
$$

that is, for $R=1$, the condition (9) is satisfied, that is, we conclude that $f(x)$ and $g(x)$ are $R$-weakly commutings, for $R=1$.
We will prove that the condition (11) is satisfied too. Since $16|x-y| /(4+x)(4+y) \leq|x-y|$, for all $x, y \geq 0$, then we have

$$
\begin{aligned}
M(g(x), g(y), \phi(t)) & =\frac{t}{t+|(4 x /(4+x))-(4 y /(4+y))|} \\
& =\frac{t}{t+(16|x-y| /(4+x)(4+y))} \\
& \geq \frac{4 t}{4 t+4|x-y|} \\
& =M\left(f(x), f(y), \phi\left(\frac{t}{c}\right)\right) .
\end{aligned}
$$

Since all the conditions of Theorem 27 are satisfied, we have that $f(x)$ and $g(x)$ have a unique common fixed point. It is easy to see that this point is $x=0$.

\section{Acknowledgments}

This paper was supported by the Ministry of Education and Science of Serbia, Project Grant no. 174032. The authors are very grateful to the referees for their helpful suggestions.

\section{References}

[1] L. A. Zadeh, "Fuzzy sets," Information and Computation, vol. 8, pp. 338-353, 1965.

[2] O. Kaleva and S. Seikkala, "On fuzzy metric spaces," Fuzzy Sets and Systems, vol. 12, no. 3, pp. 215-229, 1984.

[3] I. Kramosil and J. Michálek, "Fuzzy metrics and statistical metric spaces," Kybernetika, vol. 11, no. 5, pp. 336-344, 1975.

[4] A. George and P. Veeramani, "On some results in fuzzy metric spaces," Fuzzy Sets and Systems, vol. 64, no. 3, pp. 395-399, 1994.

[5] B. Schweizer and A. Sklar, Probabilistic Metric Spaces, Elsevier, New York, NY, USA, 1983.

[6] M. Grabiec, "Fixed points in fuzzy metric spaces," Fuzzy Sets and Systems, vol. 27, no. 3, pp. 385-389, 1988.

[7] A. George and P. Veeramani, "On some results of analysis for fuzzy metric spaces," Fuzzy Sets and Systems, vol. 90, no. 3, pp. 365-368, 1997.

[8] S. N. Ješić and N. A. Babačev, "Common fixed point theorems in intuitionistic fuzzy metric spaces and $\mathscr{L}$-fuzzy metric spaces with nonlinear contractive condition," Chaos, Solitons and Fractals, vol. 37, no. 3, pp. 675-687, 2008.

[9] S. N. Ješić, N. A. Babačev, D. O’Regan, and R. M. Nikolić, "Common fixed point theorems for four mappings defined on $L$-fuzzy metric spaces with nonlinear contractive type condition," Fixed Point Theory, vol. 10, no. 2, pp. 259-274, 2009.

[10] Y. Shen, W. Chen, and S. Wang, "A note on "Common fixed point theorems for commutating mappings in fuzzy metric spaces"', Abstract and Applied Analysis, vol. 2012, Article ID 142858, 7 pages, 2012.

[11] F. M. Zheng and X. G. Lian, "Common fixed point theorems for commutating mappings in fuzzy metric spaces," Abstract and Applied Analysis, vol. 2012, Article ID 729758, 5 pages, 2012.

[12] D. Miheț, "Altering distances in probabilistic Menger spaces," Nonlinear Analysis. Theory, Methods \& Applications A, vol. 71, no. 7-8, pp. 2734-2738, 2009. 
[13] M. S. Khan, M. Swaleh, and S. Sessa, "Fixed point theorems by altering distances between the points," Bulletin of the Australian Mathematical Society, vol. 30, no. 1, pp. 1-9, 1984.

[14] B. S. Choudhury and K. Das, "A new contraction principle in Menger spaces," Acta Mathematica Sinica (English Series), vol. 24, no. 8, pp. 1379-1386, 2008.

[15] R. P. Pant, "Common fixed points of noncommuting mappings," Journal of Mathematical Analysis and Applications, vol. 188, no. 2, pp. 436-440, 1994.

[16] R. Vasuki, "Common fixed points for $R$-weakly commuting maps in fuzzy metric spaces," Indian Journal of Pure and Applied Mathematics, vol. 30, no. 4, pp. 419-423, 1999. 


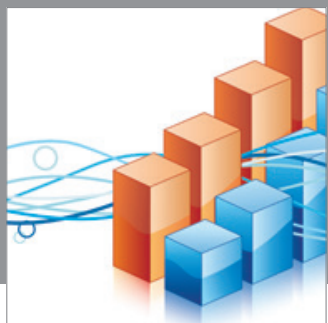

Advances in

Operations Research

mansans

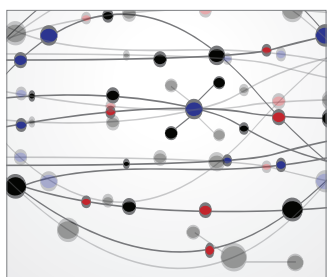

The Scientific World Journal
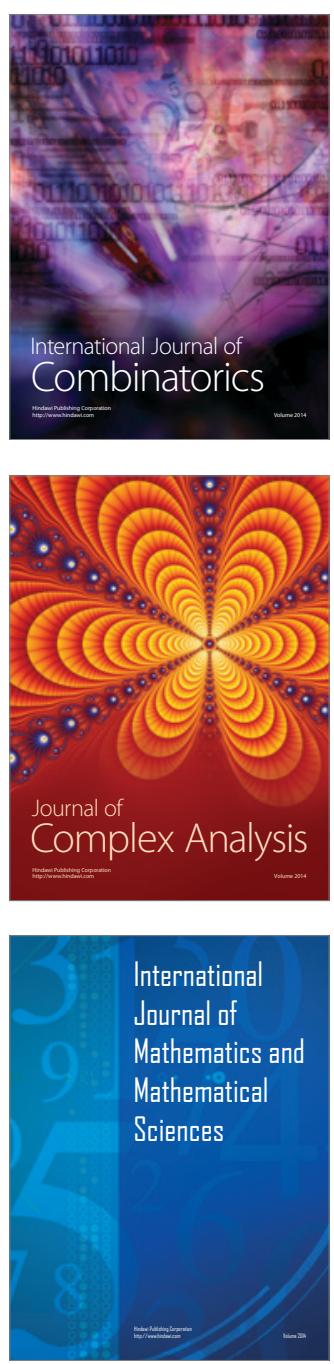
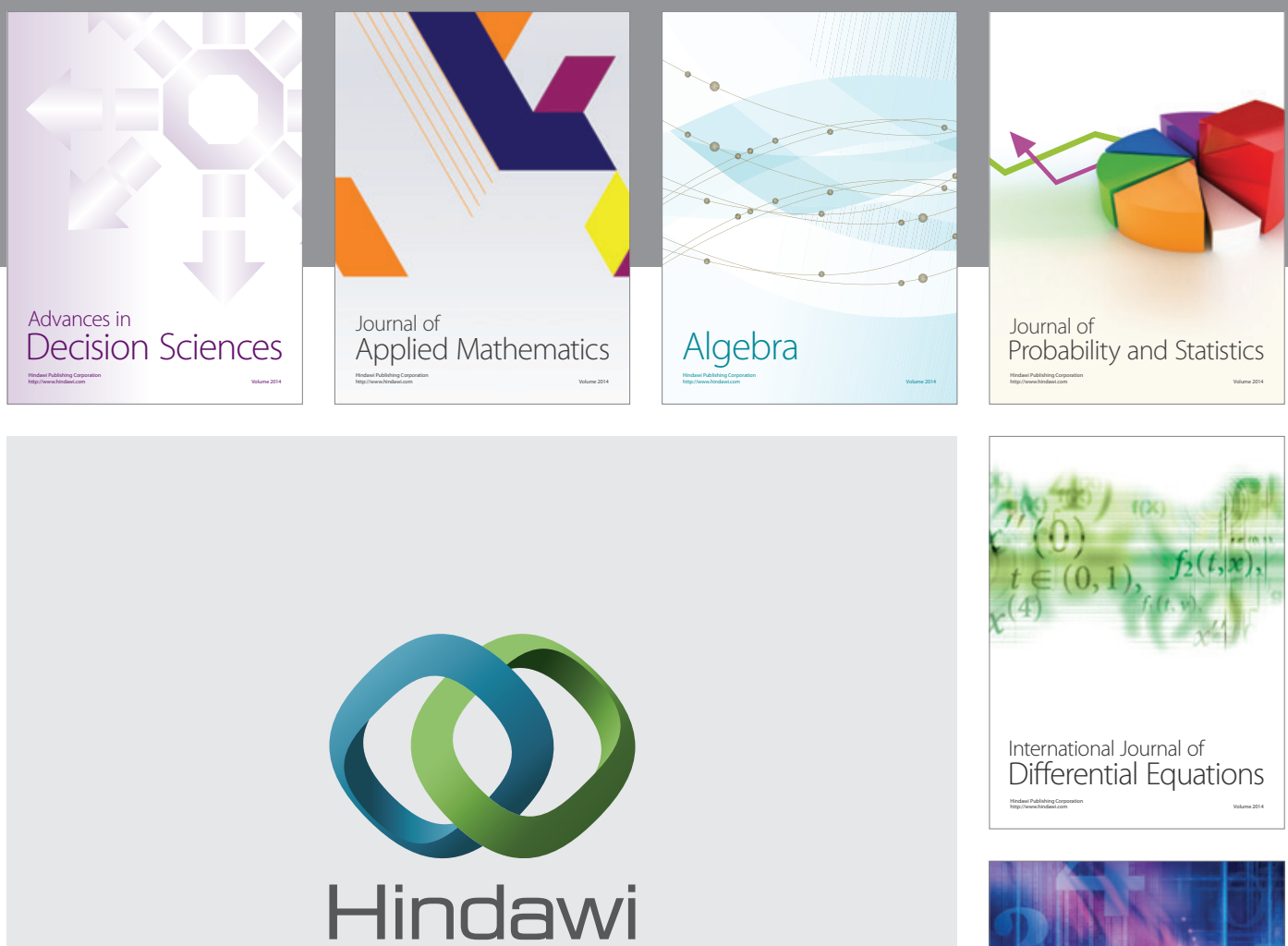

Submit your manuscripts at http://www.hindawi.com


Journal of

Function Spaces

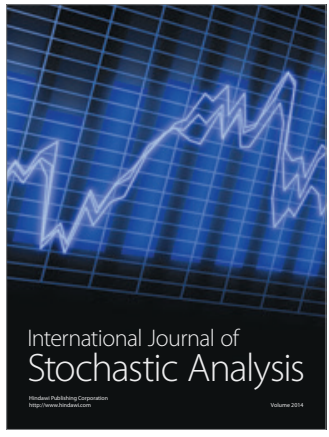

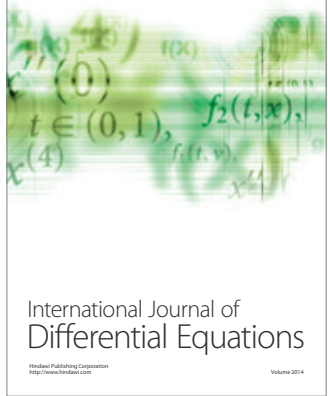
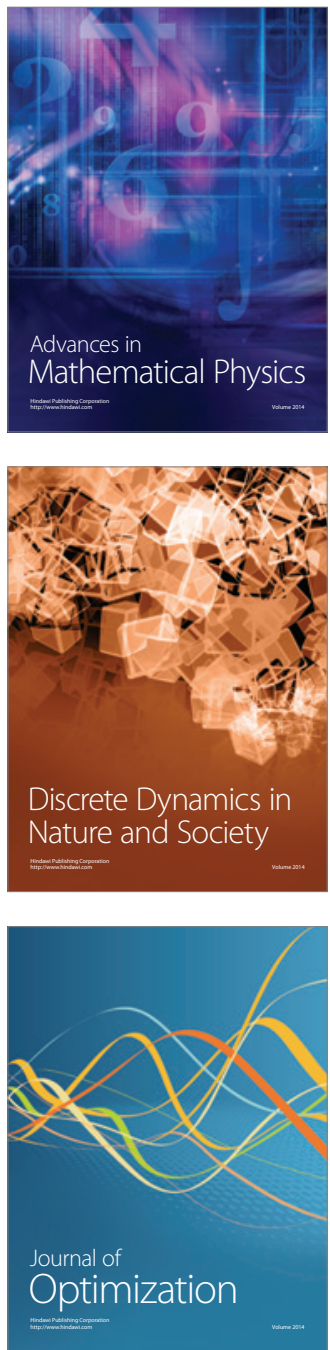